\section{A Side-Chain Dendronized Nonlinear Optical Polyimide with Large and Thermally Stable Electrooptic Activity}

\section{J ingdong Luo, Marnie Haller, Hongxiang Li, Hong-Zhi Tang, and Alex K.-Y. J en*}

Department of Material Science and Engineering, Box 352120, University of Washington,

Seattle, Washington 98195

\section{Kavitha J akka, Chia-Hung Chou, and Ching-Fong Shu}

Department of Applied Chemistry, National Chiao Tung University, Hsin-Chu, Taiwan 30035, Republic of China

Received J uly 15, 2003

Revised Manuscript Received December 4, 2003

Organic second-order nonlinear optical (NLO) materials have received increasing interests due to their potential applications in high-speed electrooptic ( $E-O)$ devices with very broad bandwidth (up to $150 \mathrm{GHz}$ ) and low driving voltages $(<1 \mathrm{~V}) .{ }^{1}$ In the pursuit of a new generation of highly efficient NLO polymers for device fabrications, we have recently developed a series of dendritic chromophores and chromophore-containing dendrimers that possess very interesting nanostructures and $\mathrm{E}-\mathrm{O}$ activities. By controlling their size, shape, and molecular architecture, we have achieved dramatically enhanced poling efficiency in these materials. ${ }^{2-6}$ These encouraging results motivate us to extend the similar site-isolation principle to several commercially available linear polymers. We have incorporated chromophores that contain fluorinated dendrons onto a hydroxylstyrene photoresist polymer to afford side-chain dendronized NLO polymers. ${ }^{7}$ This new approach allows us to achieve both high poling efficiency and reduced synthetic time for $\mathrm{E}-\mathrm{O}$ materials simultaneously. An exceptionally large $\mathrm{E}-\mathrm{O}$ coefficient (97 pm/ at $1.3 \mu \mathrm{m})$ has al so been demonstrated. This value is almost twice higher than those obtained in the guest-host systems. Even though these results are very impressive, these materials do not possess the thermal stability that is needed for practical applications. The poling-induced polar order will decay quite rapidly upon heating due to low glass-transition temperature $\left(T_{g}\right)$ of the polystyrene backbone.

To improve this deficiency, we try to apply the siteisolation concept to high-temperature polyimides. In the past decade, thermally stable and high-T $\mathrm{g}$ polyimides were vigorously pursued for fabricating $\mathrm{E}-\mathrm{O}$ devices. ${ }^{1 \mathrm{c}-\mathrm{e}, 8}$ However, most of the NLO polyimides suffer from low poling efficiency due to the intrinsic chain rigidity and $\pi-\pi$ stacking of the aromatic backbone. Usually, a hightemperature poling process is needed for orienting chromophore dipoles. As a result, only a very small number of chromophores can tolerate these stringent conditions and afford moderate optical nonlinearity. It remains a very challenging task to achieve large nonlinearity and good thermal stability simultaneously. This is also the main reason that significantly hinders the rapid deployment of $\mathrm{E}-\mathrm{O}$ devices. ${ }^{8}$

Here, we report the synthesis and characterization of a high- $\mathrm{T}_{\mathrm{g}}$ aromatic polyimide with pendant den-

\footnotetext{
* To whom correspondence should be addressed.
}

dronized NLO chromophores functionalized on a cardobisphenol linkage of a rigid aromatic polyimide backbone. Polyimides with this kind of cardo structure usually possess very high $\mathrm{T}_{\mathrm{g}}$, excellent thermal stability, and good solubility. ${ }^{9}$

Scheme 1 outlines the synthetic route for the preparation of this cardo-type polyimide $\mathbf{P I - O H}$ with pendant phenol groups. After the base hydrolysis of $\mathbf{1}$, it was further reacted with excess aniline in the presence of aniline hydrochloride to afford 9,9-bis(4-aminophenyl)2,7-dihydroxyfluorene (3). The polymerization of diamine monomer 3 with 4,4'-(hexafl uoroi sopropylidene)diphthalic anhydride was carried out in m-cresol using isoquinoline as catalyst. The resulting polyimide, PI$\mathbf{O H}$, is readily soluble in common organic solvents, such as THF, DMF, and DMSO. It was fully characterized by ${ }^{1} \mathrm{H} N M R,{ }^{13} \mathrm{C}$ NMR, GPC, and thermal analysis. The weight-average molecular weight $\left(\mathrm{M}_{\mathrm{w}}\right)$ of $\mathbf{P I - O H}$ is approximately $7.3 \times 10^{4}$ with a polydispersity index of 1.9. A distinct $\mathrm{T}_{\mathrm{g}}$ was observed at $345^{\circ} \mathrm{C}$ by differential scanning calorimetry (DSC). The high $\mathrm{T}_{\mathrm{g}}$ value of PIOH can be attributed to its rigid cardo structure along with the presence of polar hydroxy pendant groups, which in turn produce significant interchain interactions. As revealed by thermogravimetric analysis (TGA), this polymer also exhibits outstanding thermal stability with less than $5 \%$ weight loss occurring at $514{ }^{\circ} \mathrm{C}$.

The postfunctionalization process was conducted by condensing this precursor polymer with $\mathbf{R}_{\mathbf{1}} \mathbf{O H}$, the carboxylic derivatives of a dendron-capped polyene-type CLD chromophore, and a smaller pentafluorobenzoic acid, sequentially. These steps proceeded in one pot under a very mild condition using 1,3-dicyclohexylcarbodiimide (DCC) and 4-(dimethylamino)pyridinium 4-toluenesulfonate (DPTS) as the condensation reagents. The resultant NLO polyimide (PI-CLD) was fully characterized by ${ }^{1} \mathrm{H}$ NMR, ${ }^{19} \mathrm{~F}$ NMR, and UV-vis spectroscopy, GPC, and thermal analysis. It shows excellent solubility in common organic solvents, such as chloroform, tetrahydrofuran (THF), and cyclopentanone. By comparing the relative integration of aromatic and al iphatic characteristic peaks in the ${ }^{1} \mathrm{H}$ NMR spectrum, it was found that $50 \mathrm{~mol} \%$ of the phenol groups were consumed by $\mathbf{R}_{\mathbf{1}} \mathbf{O H}$. It can be converted to a $\sim 25$ wt \% loading level of the core chromophore (donor-conjugation bridgeacceptor moiety excluding the dendritic part) in PICLD. This was further proved by quantitative analysis of the UV-vis spectra of its solution in 1,4-dioxane.

It has been observed that highly polarizable pushpull compounds like CLD-type chromophores are highly solvatochromic in polar solvents and solid-state environments. For example, a CLD-type chromophore without the protection of dendritic moieties shows significant different $\lambda_{\text {max }}$ in various polymer matrices: $657 \mathrm{~nm}$ in poly(methyl methacrylate) (PMMA), $667 \mathrm{~nm}$ in amorphous polycarbonate (APC), and $698 \mathrm{~nm}$ in a polar polyquinoline (PQ-100). ${ }^{10}$ Since PI-CLD is a side-chain dendronized NLO polymer with a quite polar and rigid polyimide backbone, it should possess a quite different microenvironment to the NLO chromophores compared to that of the flexible polystyrene backbone reported earlier (F igure 1 inset). ${ }^{7}$ However, even such a different macromolecular environment, PI-CLD shows a very similar chromophoric charge-transfer absorption peak 
Scheme 1. Synthesis of Cardo-Type NLO Polyimide (PI-CLD) with Dendronized Chromophore

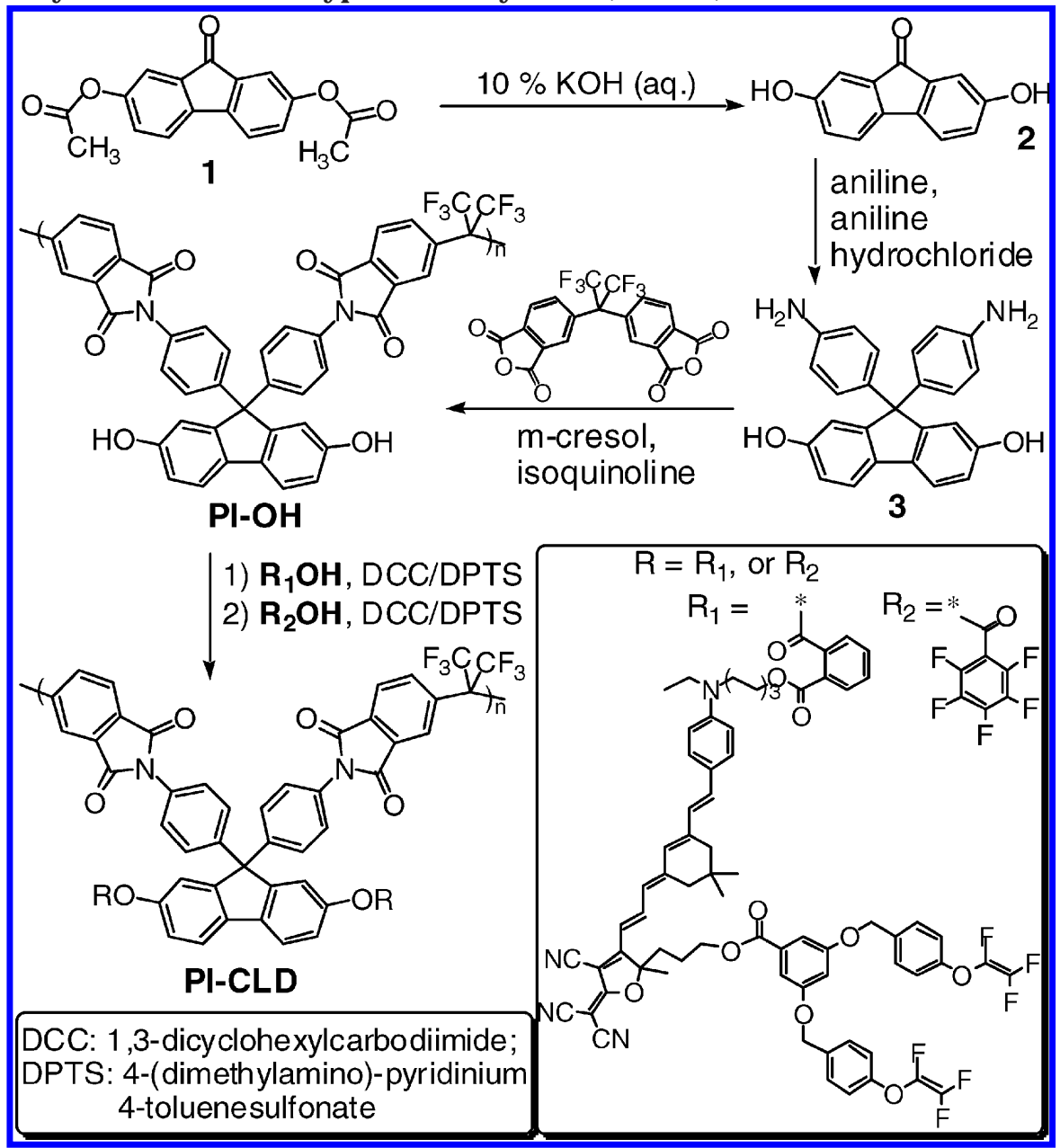

Table 1. Physical Property Comparison between Two Side-Chain Dendronized NLO Polymers PI-CLD and PS-CLD

\begin{tabular}{|c|c|c|c|c|c|c|c|c|}
\hline & $\begin{array}{l}\lambda_{\max } \\
(\mathrm{nm})\end{array}$ & $\begin{array}{c}\mathrm{n}_{1300}{ }^{\mathrm{a}} \\
\left(\mathrm{n}_{\mathrm{TE}} / \mathrm{n}_{\mathrm{TM}}\right)\end{array}$ & $\begin{array}{c}n_{1550} b \\
\left(n_{T E} / n_{T M}\right)\end{array}$ & $\mathrm{T}_{\mathrm{g}}\left({ }^{\circ} \mathrm{C}\right)$ & $\begin{array}{l}\text { dye content } \\
\text { (wt \%) }\end{array}$ & $\begin{array}{l}\text { poling field } \\
(\mathrm{V} / \mu \mathrm{m})\end{array}$ & $r_{33}{ }^{c}(p m / N)$ & $\begin{array}{c}\text { poling efficiency }(\%)^{d} \\
\left(r_{\text {actual }} / r_{\text {theo }}\right)\end{array}$ \\
\hline $\begin{array}{l}\text { PI-CLD } \\
\text { PS-CLD }\end{array}$ & $\begin{array}{l}711 \\
708\end{array}$ & $\begin{array}{l}1.7104 / 1.6864 \\
1.5851 / 1.5776\end{array}$ & $\begin{array}{l}1.6875 / 1.6660 \\
1.5813 / 1.5738\end{array}$ & $\begin{array}{r}155 \\
90\end{array}$ & $\begin{array}{l}25 \\
20\end{array}$ & $\begin{array}{r}85 \\
142\end{array}$ & $\begin{array}{l}71 \\
97\end{array}$ & $\begin{array}{l}81 \\
78\end{array}$ \\
\hline
\end{tabular}

a The refractive indexes at $1.3 \mu \mathrm{m}$. ${ }^{\mathrm{b}}$ The refractive indexes at $1.55 \mu \mathrm{m}$. ${ }^{\mathrm{c}}$ The experimental EO coefficient at $1.3 \mu \mathrm{m}$. ${ }^{\mathrm{d}}$ The ratio of experimental $r_{33}$ value ( $\left.r_{\text {actual }}\right)$ to theoretically predicted $r_{33}$ value $\left(r_{\text {theo }}\right)$ using the two-level model.

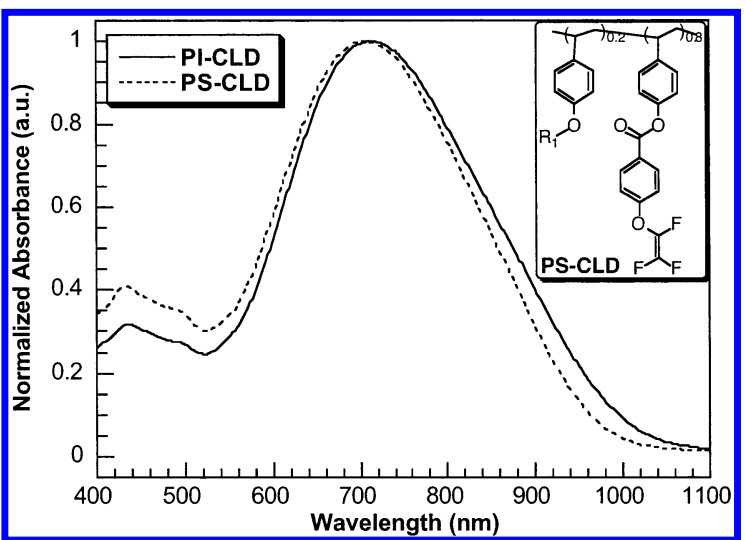

Figure 1. UV-vis spectrum comparison of two side-chain dendronized NLO polymers in different polymer matrixes: PICLD, polyimide; PS-CLD, polystyrene.

compared to that of PS-CLD (Figure 1). The UV-vis spectra of the thin film PI-CLD and PS-CLD on glass slides exhibit similar main absorption bands with absorption maxima $\left(\lambda_{\max } \mathrm{s}\right)$ at 711 and $708 \mathrm{~nm}$, respectively, both of which are ascribed to the $\pi-\pi^{*}$ charge- transfer band of the CLD chromophore (Table 1). This comparison demonstrates that the environment around the chromophores is dictated by the site-isolation effect provided by the surrounding dendrons and is independent of the polymer matrix that we selected. This finding is also in good agreement with the similar solvatochromism study of the 3-D shape dendritic chromophore that we previously reported. ${ }^{3}$

The $\mathrm{E}-\mathrm{O}$ measurements for PI-CLD, and the detailed comparisons of its poling parameters with those of PS-CLD, are critical for evaluating the role of the dendrons in affecting the poling efficiency of PI-CLD. The solution of PI-CLD in cyclopentanone (11 wt \%, filtered through a $0.2 \mu \mathrm{m}$ syringe filter) was spin-coated onto indium tin oxide (ITO) gl ass substrates. The films were baked under vacuum at $100^{\circ} \mathrm{C}$ overnight to ensure the removal of the residual solvent. Then a thin layer of gold was sputtered onto the films as the top el ectrode for performing the high-electric field poling. The optimum poling condition of the film was achieved at 155 ${ }^{\circ} \mathrm{C}$ with a DC electric field of $0.85 \mathrm{MV} / \mathrm{cm}$. The $r_{33}$ value was measured using the thin-film reflection technique at $1.3 \mu \mathrm{m}^{11}$ and showed a very large $\mathrm{E}-\mathrm{O}$ coefficient of 


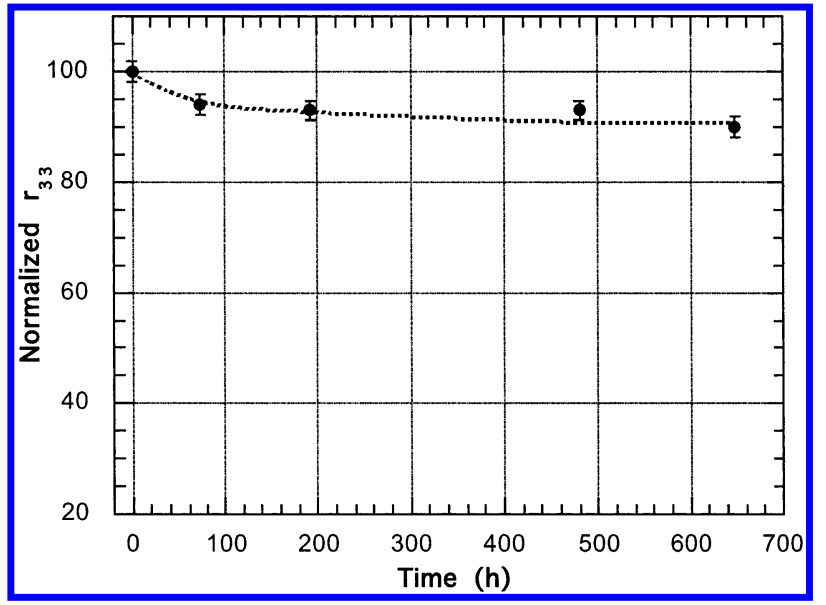

Figure 2. Temporal stability of $\mathrm{E}-\mathrm{O}$ activity of PI-CLD at $85^{\circ} \mathrm{C}$.

$71 \mathrm{pm} N$. This high $r_{33}$ value represents $\sim 81 \%$ of the highest achievable $r_{33}$ value $(88 \mathrm{pm} / \mathrm{N})$ that is predicted by using the two-level model. ${ }^{12}$ Most importantly, when compared to the flexible polymer PS-CLD, the similar high poling efficiency ( $~ 80 \%$ of the theoretically achievable $\mathrm{E}-\mathrm{O}$ activity) has been reproduced in high $\mathrm{T}_{\mathrm{g}}$ and rigid polymer PI-CLD.

Moreover, this high-Tg PI-CLD exhibits a much improved alignment stability compared to that of PSCLD. The poled PI-CLD retains higher than $90 \%$ of its original $r_{33}$ value after more than $600 \mathrm{~h}$ at $85^{\circ} \mathrm{C}$ (Figure 2). For comparison, the flexible polymer PSCLD with a much lower $\mathrm{T}_{\mathrm{g}}\left(90^{\circ} \mathrm{C}\right)$ shows only $37 \%$ of its original $r_{33}$ value after heating at $70{ }^{\circ} \mathrm{C}$ for $144 \mathrm{~h}$.

The structural features of PI-CLD can explain very well for its high poling efficiency and excellent thermal stability. This cardo-type polyimi de has a rigid and 3-D extended main chain, and along the $\mathrm{V}$-bended repeating units, the dendronized chromophores are attached on the fluorene ring that is perpendicular to the imido backbone. This kind of spatial arrangement can suppress strong interchain interactions of polyimide and reduce potential phase separation between the highly aromatic, rigid polyimide backbone and chromophores. In addition, the cardo-type linkage of the dendronized chromophores may also facilitate the self-assembly of these NLO molecules to form potentially ordered cylindrical rigid rods which will facilitate the dipole alignment during the poling process because less steric hindrance caused by chromophore/polymer chain entanglements.

In conclusion, we have successfully applied the siteisolation principle to a rigid 3-D cardo-type polyimide with very high $T_{g}$. High poling efficiency has been achieved to afford a very large $\mathrm{E}-\mathrm{O}$ coefficient (71 pm $\mathrm{N}$ at $1.3 \mu \mathrm{m})$. More than $90 \%$ of this value can be retained at $85^{\circ} \mathrm{C}$ for more than $650 \mathrm{~h}$. Comprehensive studies to optimize the properties of these high-T $\mathrm{g}$ materials for $\mathrm{E}-\mathrm{O}$ device fabrication are in progress.
Acknowledgment. Financial support from the National Science Foundation (NSF-NIRT and the NSFSTC Program under Agreement DMR-0120967) and the Air Force Office of Scientific Research (AFOSR) under the MURI Center on Polymeric Smart Skins is acknowledged. Alex K.-Y. J en thanks the Boeing-J ohnson Foundation for its support.

Supporting Information Available: Text giving detailed experimental information for material preparation and structural characterization. This material is available free of charge via the Internet at http://pubs.acs.org.

\section{References and Notes}

(1) Shi, Y.; Zhang, C.; Zhang, H.; Bechtel, J . H.; Dalton, L. R.; Robinson, B. H.; Steier, W. H. Science 2000, 288, 119. (b) Lee, M.; Katz, H. E.; Erben, C.; Gill, D. M.; Gopalan, P.; Heber, J. D.; McGee, D. L. Science 2002, 298, 1401. (c) Marder, S. R.; Kippelen, B.; J en, A. K.-Y.; Peyghambarian, N. Nature (London) 1997, 388, 845. (d) Marks, T. J .; Ratner, M. A. Angew. Chem., Int. Ed. Enql. 1995, 34, 155. (e) Burland, D. M.; Miller, R. D.; Walsh, C. A. Chem. Rev. 1994 94, 31. (f) Kajzar, F.; Lee, K.-S.; J en, A. K.-Y. Adv. Polym. Sci. 2003, 161, 1 .

(2) Ma, H.; Liu, S.; Luo, J .; Suresh, S.; Liu, L.; Kang, S. H.; Haller, M.; Sassa, T.; Dalton, L. R.; J en, A. K.-Y. Adv. Funct. Mater. 2002, 12, 565.

(3) Luo, J.; Ma, H.; Haller, M.; J en, A. K.-Y.; Barto, R. R. Chem. Commun. 2002, 888.

(4) Robinson, B. H.; Dalton, L. R.; Harper, H. W.; Ren, A.; Wang, F.; Zhang, C.; Todorova, G.; Lee, M.; Aniszfeld, R.; Garner, S.; Chen, A.; Steier, W. H.; Houbrecht, S.; Persoons, A.; Ledoux, I.; Zyss, J.; J en, A. K.-Y. Chem. Phys. 1999, 245 35. (b) Robinson, B. H.; Dalton, L. R. L. Phys. Chem. A 2000, 104, 4785. (c) Dalton, L. R.; Steier, W. H.; Robinson, B. H.; Zhang, C.; Ren, A.; Garner, S.; Chen, A.; Londergan, T.; Irwin, L.; Carlson, B.; Fifield, L.; Phelan, G.; Kincaid, C.; Amend, J .; J en, A. K.-Y. L. Mater. Chem. 1999, 9, 19.

(5) Ma, H.; J en, A. K.-Y. Adv. Mater. 2001, 13, 1201.

(6) Ma, H.; Chen, B. Q.; Sassa, T.; Dalton, L. R.; J en, A. K.-Y. L. Am. Chem. Soc. 2001, 123, 986.

(7) Luo, J.; Liu, S.; Haller, M.; Lu, L.; Ma, H.; J en, A. K.-Y. Adv. Mater. 2002, 14, 1763.

(8) Verbiest, T.; Burland, D. M.; J urich, M. C.; Lee, V. Y.; Miller, R. D.; Volksen, W. Science 1995, 268, 1604. (b) Saadeh, H. Yu, D.; Wang, L. M.; Yu, L. P. L. Mater. Chem. 1999, 9, 1865. (c) Chen, T. A.; J en, A. K.-Y.; Cai, Y. M. L. Am. Chem. Soc. 1995, 117, 7295. (d) Davey, M. H.; Lee, V. Y.; Wu, L.-M.; Moylan, C. R.; Volksen, W.; Knoesen, A.; Miller, R. D.; Marks, T. L. Chem. Mater. 2000, 12, 1679.

(9) Korshak, V. V.; Vinogradova, S. V.; Vygodskii, Y. S. L. Macromol. Sci., Rev. Macromol. Chem. Part C 1974, 11, $4 \overline{5}$. (b) Yang, C. P.; Lin, J. H. L. Polym. Sci., Part A: Polym. Chem. 1993, 31, 2153. (c) Hsiao, S. H.; Li, C. T. L. Polym. Sci., Part A: Polym. Chem. 1999, 37, 1403.

(10) Zhang, C.; Dalton, L. R.; Öh, M.-C.; Zhang, H.; Steier, W. H. Chem. Mater. 2001, 13, 3043. (b) Liakatas, I.; Cai, C.; Bosch, M.; J ager, M.; Bosshard, Ch.; Gunter, P.; Zhang, C.; Dalton, L. R. Appl. Phys. Lett. 2000, 76, 1368.

(11) Teng, C. C.; Man, H. T. Appl. Phys. Lett. 1990, 56, 1734.

(12) Katz, H. E.; Dirk, C. W.; Schilling, M. L.; Singer, K. D.; Sohn, J. E. Mater. Res. Soc. Symp. Proc. 1987, 109, 127. The $r_{33}$ value at $1.55 \mu \mathrm{m}$ is $58 \mathrm{pm} / \mathrm{N}$ estimated from the same twolevel model using the experimental value of $71 \mathrm{pm} / \mathrm{at} 1300$ $\mathrm{nm}$.

MA0350009 